\title{
Thunderclap headache and reversible segmental cerebral vasoconstriction associated with use of oxymetazoline nasal spray
}

\author{
Andrea H.S. Loewen, Mark E. Hudon, Michael D. Hill
}

\section{Abstract}

OXYMETAZOLINE IS A SYMPATHOMIMETIC amine found in over-thecounter nasal decongestants. We report a case of chronic use of nasal oxymetazoline associated with thunderclap headache due to reversible segmental intracranial vasoconstriction.

CMAJ 2004;171(6):593-4

A 31-year-old woman had sudden onset of global headache at $1 \mathrm{pm}$ when she was resting that she characterized as the "worst of her life," rating the pain as 10/10. Associated symptoms included nausea, vomiting, sonophobia and photophobia. The headache waxed and waned over the next 4 days, and on day 4 the patient presented to the emergency department in a community hospital. Her blood pressure, findings on neurological examination and initial CT scan were normal. Her past medical history included hiatal hernia, cigarette smoking and remote use of marijuana (she denied any other illicit drugs), and she was taking sertraline, peptobismol, lansoprazole and domperidone. A lumbar puncture revealed normal cerebrospinal fluid with no xanthochromia. The opening pressure in the seated position was $37 \mathrm{~cm} \mathrm{H}_{2} \mathrm{O}$. She was transferred to our hospital, and CT venography was performed. It revealed no venous sinus thrombosis, but multifocal vessel irregularities in both the anterior and posterior arterial circulations were observed. Cerebral angiography confirmed focal areas of vasospasm in the internal carotid circulations bilaterally as well as in the vertebrobasilar system (Fig. 1A, B, C). Results of hematologic and serologic investigations were negative for signs of systemic infection, inflammation or vasculitis.

The patient had been using Afrin, a nasal spray that contains oxymetazoline, regularly for the previous 6 months. Although she was using the medication at recommended daily dosages (2-3 sprays twice daily), she was using it consistently. Two weeks before presentation she had noticed a pattern of headache starting 20 minutes after use of the nasal spray. The index event had occurred immediately after its use.

Use of the nasal spray was discontinued. Narcotic analgesics reduced the pain, but the nausea and vomiting re- sponded to ondansetron only. The patient had no improvement in her headache with nimodipine, a calcium-channel antagonist that causes dilatation of arterial smooth muscle. Repeat lumbar puncture was done in the supine position at discharge and demonstrated an opening pressure of $19 \mathrm{~cm}$ $\mathrm{H}_{2} \mathrm{O}$. Two weeks after discharge the headache had nearly resolved. A repeat angiogram at 6 weeks showed complete resolution of most areas of arterial narrowing (Fig. 1D).

\section{Comments}

Thunderclap headache is a hyperacute, severe headache that is so named because of its sudden onset. It often heralds a serious intracranial vascular event such as subarachnoid hemorrhage, cerebral venous sinus thrombosis or pituitary apoplexy. However, it may also occur spontaneously or in the setting of cerebral vasoconstriction. Duration may be from 1 hour to 10 days. ${ }^{1}$ In this case, based on angiographic follow-up, the underlying cause of thunderclap headache was found to be reversible segmental cerebral vasoconstriction.

Cerebral vasoconstriction has been reported with cerebral vascular events, especially subarachnoid hemorrhage; as a complication of disease states such as porphyric encephalopathy, pheochromocytoma and eclampsia; and as a result of exogenously administered blood products or drugs such as intravenous immunoglobulin in Guillain-Barré syndrome, angiographic contrast medium, amphetamines, serotonergic drugs and methylenedioxymethamphetamine (ecstasy) ${ }^{1,2}$ The patient in our case was taking sertraline concurrently with oxymetazoline. Selective serotonin reuptake inhibitors have been associated with reversible segmental cerebral vasoconstriction and stroke. The patient's headache improved after cessation of the oxymetazoline alone, and thus it appears to have been the primary agent in her cerebral vasoconstriction. However, an interaction with sertraline could also have played a role.

Oxymetazoline is a selective $\alpha_{2 \mathrm{~A}}$-adrenergic receptor agonist. It is used as a topical vasoconstrictor for rhinitis. Adverse effects include rebound congestion, hypertension, palpitations and headaches. There have been case reports of 


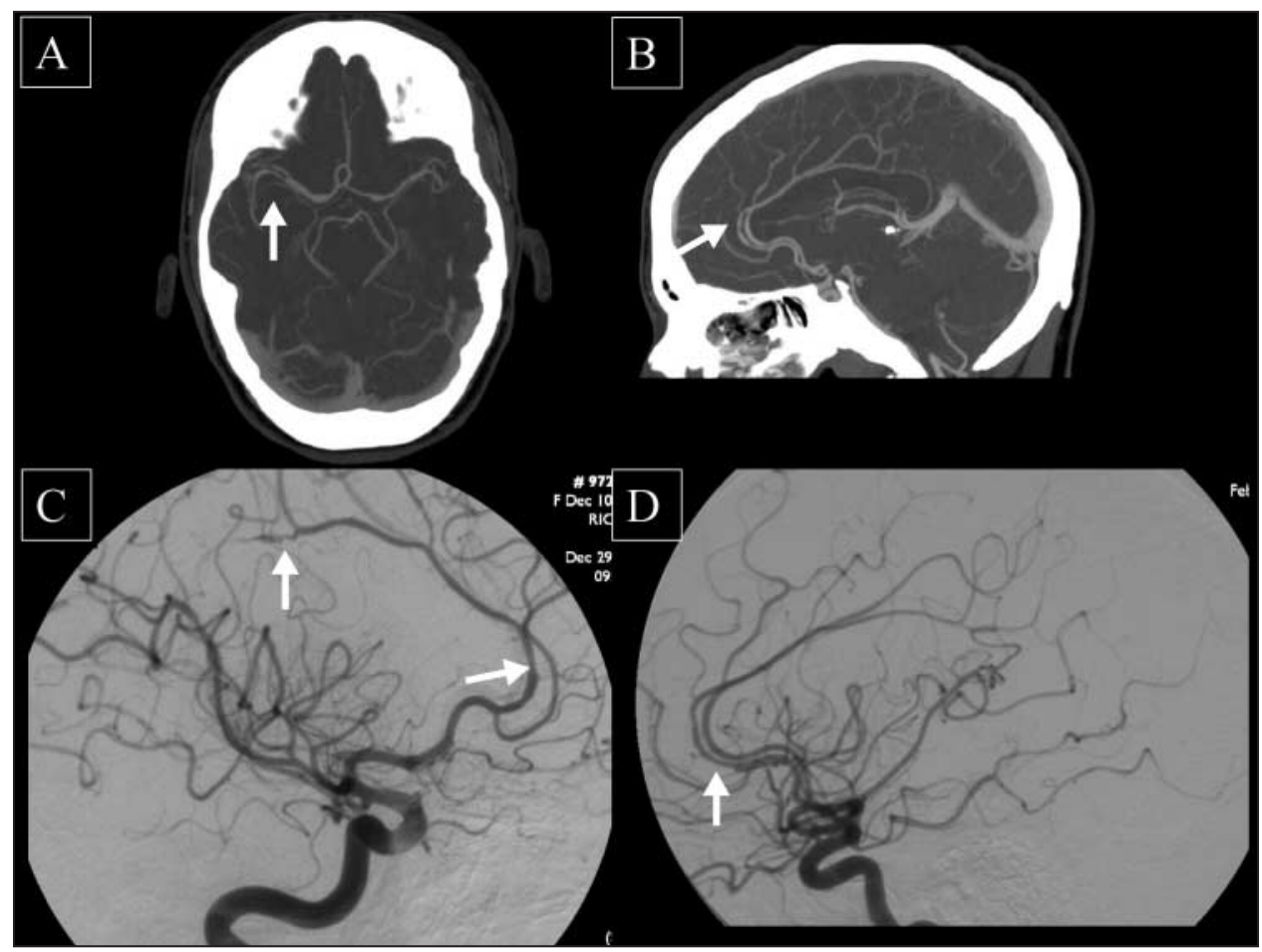

Fig. 1: A: CT angiogram at admission showing axial reformatted images with severe narrowing of the $\mathrm{M} 2$ branches of the right middle cerebral artery. B: CT angiogram at admission showing midline sagittal multiplanar reformatted image; done to assess dural sinuses, the image shows multifocal narrowings of the anterior cerebral arteries. C: Right anterior oblique view from a selective cerebral angiogram of the right internal carotid artery showing focal narrowing and dilatation of anterior cerebral artery. D: Follow-up left anterior oblique view from the left carotid artery showing near complete resolution of arteriopathic changes.

stroke associated with oxymetazoline, but none described cerebral vasoconstriction. ${ }^{3,4}$ This case report illustrates that oxymetazoline can cause reversible segmental cerebral vasoconstriction, resulting in thunderclap headache.

Possible mechanisms of stroke with sympathomimetics include acute hypertension, hemorrhage, vasospasm, vasospasm-induced thrombosis and angiitis. In this case, because of the reversible changes, we believe the patient had cerebral vasospasm, but a concurrent arteritis is possible given the long duration of exposure. Since resolution of this arteritis with cessation of the drug would be expected, the patient will be monitored for recurrence of clinical symptoms.

This case highlights a potential systemic toxic effect of oxymetazoline nasal spray, specifically reversible segmental cerebral vasoconstriction causing thunderclap headache. Patients presenting with thunderclap headache should be questioned about their use of predisposing medications including over-the-counter nasal decongestants. In addition to cerebral angiography, CT and MRI angiography and transcranial doppler have been used to diagnose intracerebral vasoconstriction in other cases. Abstinence from the offending drug is the main therapy, although calciumchannel blockers may also have a therapeutic role.
This article has been peer reviewed.

From the Departments of Medicine (Loewen, Hill), Radiology (Hudon), Clinical Neurosciences (Hudon, Hill) and Community Health Sciences (Hill), Faculty of Medicine, University of Calgary, Calgary, Alta.

Competing interests: None declared.

Contributors: Andrea Loewen wrote the first draft of the manuscript with input and editorial assistance from Mark Hudon and Michael Hill. All authors approved the final manuscript for publication.

\section{References}

1. Dodick DW, Brown Jr RD, Britton JW, Huston III J. Nonaneurysmal thunderclap headache with diffuse, multifocal, segmental, and reversible vasospasm. Cephalalgia 1999;19:118-23.

2. Sturm JW, Macdonell RAL. Recurrent thunderclap headache associated with reversible intracerebral vasospasm causing stroke. Cephalalgia 2000;20:132-5.

3. Montalban J, Ibanez L, Rodriguez C, Lopez M, Sumalla J, Codina A. Cerebral infarction after excessive use of nasal decongestants. 7 Neurol Neurosurg Psychiatry 1989;52:541-3.

4. Cantu C, Arauz A, Murillo-Bonilla LM, Lopez M, Barinagarrementeria F. Stroke associated with sympathomimetics contained in over-the-counter cough and cold drugs. Stroke 2003;34(7):1667-73.

Correspondence to: Michael D. Hill, Assistant Professor, Calgary

Stroke Program, Department of Clinical Neurosciences,

University of Calgary, Foothills Hospital, Rm. 1242A,

1403-29th St. NW, Calgary AB T2N 2T9; fax 403 283-2270;

michael.hill@calgaryhealthregion.ca 